\title{
Resonance theory of the crossover from Bardeen-Cooper- Schrieffer superfluidity to Bose-Einstein condensation in a dilute Fermi gas
}

Citation for published version (APA):

Milstein, J. N., Kokkelmans, S. J. J. M. F., \& Holland, M. J. (2002). Resonance theory of the crossover from Bardeen-Cooper-Schrieffer superfluidity to Bose-Einstein condensation in a dilute Fermi gas. Physical Review $A$ : Atomic, Molecular and Optical Physics, 66(4), 043604-1/6. [043604].

https://doi.org/10.1103/PhysRevA.66.043604

DOI:

10.1103/PhysRevA.66.043604

Document status and date:

Published: 01/01/2002

Document Version:

Publisher's PDF, also known as Version of Record (includes final page, issue and volume numbers)

Please check the document version of this publication:

- A submitted manuscript is the version of the article upon submission and before peer-review. There can be important differences between the submitted version and the official published version of record. People interested in the research are advised to contact the author for the final version of the publication, or visit the $\mathrm{DOI}$ to the publisher's website.

- The final author version and the galley proof are versions of the publication after peer review.

- The final published version features the final layout of the paper including the volume, issue and page numbers.

Link to publication

\footnotetext{
General rights

- You may freely distribute the URL identifying the publication in the public portal. follow below link for the End User Agreement:

www.tue.nl/taverne

Take down policy

If you believe that this document breaches copyright please contact us at:

openaccess@tue.nl

providing details and we will investigate your claim.
}

Copyright and moral rights for the publications made accessible in the public portal are retained by the authors and/or other copyright owners and it is a condition of accessing publications that users recognise and abide by the legal requirements associated with these rights.

- Users may download and print one copy of any publication from the public portal for the purpose of private study or research.

- You may not further distribute the material or use it for any profit-making activity or commercial gain

If the publication is distributed under the terms of Article $25 \mathrm{fa}$ of the Dutch Copyright Act, indicated by the "Taverne" license above, please 


\title{
Resonance theory of the crossover from Bardeen-Cooper-Schrieffer superfluidity to Bose-Einstein condensation in a dilute Fermi gas
}

\author{
J. N. Milstein, S. J. J. M. F. Kokkelmans, and M. J. Holland \\ JILA, University of Colorado and National Institute of Standards and Technology, Boulder, Colorado 80309-0440
}

(Received 15 April 2002; revised manuscript received 19 July 2002; published 10 October 2002)

\begin{abstract}
We present a description of the behavior of a superfluid gas of fermions in the presence of a Feshbach resonance over the complete range of magnetic field detunings. Starting from a resonance Hamiltonian, we exploit a functional method to describe the continuous behavior from Bardeen-Cooper-Schrieffer to BoseEinstein condensation type superfluidity. Our results show an ability for a resonance system to exhibit a high critical temperature comparable to the Fermi temperature. The results are derived in a manner that is shown to be consistent with the underlying microscopic scattering physics.
\end{abstract}

DOI: 10.1103/PhysRevA.66.043604

\section{INTRODUCTION}

The ability to cool a gas of fermionic atoms well into the regime of quantum degeneracy hints at the exciting possibility of allowing one to study the mechanisms of superfluidity in an entirely new context [1]. Since these systems remain extremely dilute and are not complicated by long-range Coulomb interactions or lattice effects, cold degenerate gases seem ideal for the study of the fundamental physics behind the exotic behavior of superfluidity. Unfortunately, temperatures of around $0.2 T_{F}$ are the current state of the art in cooling [1-6]. To obtain such high critical temperatures a strong coupling mechanism is required forcing the theoretical description to extend beyond the standard Bardeen-CooperSchrieffer (BCS) approach.

Several theoretical papers have studied the effects of increasing the two-particle interactions [7-9], characterizing the coupling processes by large negative scattering lengths. We have focused in detail upon a related yet distinct approach [10], which is to significantly increase the interatomic couplings by making use of a Feshbach resonance. The difference is that, in the neighborhood of the resonance, the interactions can no longer be adequately described by a scattering length, since the scattering length diverges as one approaches the resonance. This is an artifact of the approximations made in formulating the theory since the full energydependent scattering T-matrix, which is the true descriptor of the two-particle interactions, does not diverge at finite scattering energy. This leads us to explicitly incorporate the physics of the resonance into our microscopic description of the interatomic couplings. A much more detailed discussion of this can be found in Ref. [10].

So far, we have not discussed in detail the role of fluctuations, which can have a significant effect on the critical behavior [11]. How we incorporate these fluctuations proves crucial in describing the physics correctly within the crossover regime where we find a significant population of tightly bound composite particles. The aim, therefore, of this paper will be to account for fluctuations in such a way as to properly describe the behavior of a superfluid Fermi gas at all detunings from the resonance. Recently, a complimentary treatment was independently developed by Ohashi and Griffin [13]. The slight quantitative differences between their results for the critical superfluid transition temperature and the
PACS number(s): 03.75.Fi, 64.60.-i, 74.20.-z

values we will present here appear to arise primarily from the use of quite different two-body scattering parameters and a distinct renormalization procedure.

The problem of describing a superfluid Fermi gas at all coupling strengths has been extensively studied in recent years, motivated by a desire to explain the properties of "exotic" high- $T_{c}$ superconductors, whose behavior seems to lie in a region somewhere between BCS superconductivity and Bose-Einstein condensation (BEC). An early description of the crossover from BCS to BEC superconductivity was put forth by Nozières and Schmitt-Rink (NSR) [14], after the pioneering work of Eagles [15] and Leggett [16], and later expanded upon by various authors [17-19]. A functional analysis of the crossover behavior, which is the method that we will employ, was equated to the NSR method by Randeria et al. [19]. We will adapt this method to a resonant system as necessary to describe the relevant physics of superfluidity in dilute atomic gases. It should be stressed that our method contains the multichannel interatomic couplings intrinsic to the Feshbach resonance. This was not considered in previous calculations in the context of condensed matter systems.

\section{RESONANT ACTION}

We consider the Feshbach resonance [20] for $s$-wave scattering of atoms in the lowest two hyperfine states of a fermionic alkali atom, denoted symbolically by $\sigma \in\{\uparrow, \downarrow\}$. For a homogeneous system we have the following generalized Hamiltonian:

$$
\begin{aligned}
\hat{H}(t)= & \sum_{\sigma} \int \psi_{\sigma}^{\dagger}(x)\left(\hat{H}_{\sigma}-\mu\right) \psi_{\sigma}(x) d^{3} \boldsymbol{x} \\
& +\sum_{j} \int \psi_{m_{j}}^{\dagger}(x)\left(\hat{H}_{m_{j}}-2 \mu+\nu_{j}\right) \psi_{m_{j}}(x) d^{3} \boldsymbol{x} \\
& +\int U\left(\boldsymbol{x}-\boldsymbol{x}^{\prime}\right) \psi_{\uparrow}^{\dagger}(x) \psi_{\downarrow}^{\dagger}\left(x^{\prime}\right) \psi_{\downarrow}\left(x^{\prime}\right) \psi_{\uparrow}(x) d^{3} \boldsymbol{x} d^{3} \boldsymbol{x}^{\prime} \\
& +\sum_{j} \int\left[g_{j}\left(\boldsymbol{x}-\boldsymbol{x}^{\prime}\right) \psi_{m_{j}}^{\dagger}\left(\frac{x+x^{\prime}}{2}\right) \psi_{\downarrow}(x) \psi_{\uparrow}\left(x^{\prime}\right)\right. \\
& + \text { H.c. }] d^{3} \boldsymbol{x} d^{3} \boldsymbol{x}^{\prime},
\end{aligned}
$$


where the operators $\psi_{\sigma}^{\dagger}\left(\psi_{\sigma}\right)$ create (annihilate) fermions at $x=(\boldsymbol{x}, t)$, and $\psi_{m_{j}}^{\dagger}\left(\psi_{m_{j}}\right)$ create (annihilate) composite bosons. The free dispersion Hamiltonian for fermions (bosons) is $\hat{H}_{\sigma}\left(\hat{H}_{m_{j}}\right)$ and $\nu_{j}$ is the detuning of the $j$ th molecular state from the collision continuum. The collisional interactions are described by both background fermion scattering $(U)$ and an interconversion between composite bosons and fermion pairs $\left(g_{j}\right)$.

Functional methods prove to be especially convenient in describing the thermodynamics of the resonant system. For a finite temperature field theory, the connection with statistical mechanics is made by Wick rotating the time coordinate $t \rightarrow-i \tau$ so that one works in terms of the spatial coordinate $x$ and temperature $\tau$ [21]. In this space, we define the action in the usual way,

$$
S=\sum_{l} \int_{0}^{\beta} d \tau \int d^{3} \boldsymbol{x} \psi_{l}^{\dagger}(\boldsymbol{x}, \tau) \partial_{\tau} \psi_{l}(\boldsymbol{x}, \tau)-\int_{0}^{\beta} \hat{H}(\tau) d \tau,
$$

where the sum in $l$ runs over both the Fermi and the Bose degrees of freedom. In this functional formulation we treat the fermion fields $\psi_{\sigma}$ as Grassmann variables [22] and the composite Bose fields $\psi_{m_{j}}$ as classical fields.

Let us consider a system comprised of fermions at some finite temperature $\tau$ inside a box of volume $V$ (for convenience, let us work in the set of units where $\hbar=k_{b}=1$ ). By imposing periodic boundary conditions upon the fields $\psi_{\sigma}$ and $\psi_{m_{j}}$, we form the following Fourier series expansions

$$
\begin{aligned}
& \psi_{\sigma}(\boldsymbol{x}, \tau)=(\beta V)^{-1 / 2} \sum_{\boldsymbol{k}, \omega} e^{i(\omega \tau+\boldsymbol{p} \cdot \boldsymbol{x})} a_{\sigma}(p), \\
& \psi_{m_{j}}(\boldsymbol{x}, \tau)=(\beta V)^{-1 / 2} \sum_{\boldsymbol{q}, v} e^{i(v \tau+\boldsymbol{q} \cdot \boldsymbol{x})} b_{j}(q),
\end{aligned}
$$

with even thermal (Matsubara) frequencies for the bosons $(v=2 \pi n / \beta$, where $n$ is an integer) and odd frequencies for the fermions $[\omega=2 \pi(n+1) / \beta]$, to preserve the particle statistics. Here $a_{\sigma}(p)$ annihilates a fermion at $p=(\boldsymbol{k}, \omega)$ and $b_{j}(q)$ annihilates a molecule at $\mathbf{q}=(\boldsymbol{q}, \omega)$.

By making use of the above transformation, Eq. (3), we may write out the action for the resonant system in terms of the Fourier coefficients $a_{\sigma}(p)$ and $b_{j}(q)$. In order to help clarify the following calculation, we split the resulting resonant action into two parts, the first being the usual BCS action:

$$
\begin{aligned}
S_{\mathrm{BCS}}= & \sum_{p, \sigma}\left(i \omega-\frac{p^{2}}{2 m}+\mu\right) a_{\sigma}^{*}(p) a_{\sigma}(p) \\
& -\frac{1}{\beta V} \sum_{p_{1}+p_{2}=p_{3}+p_{4}} U a_{\uparrow}^{*}\left(p_{1}\right) a_{\downarrow}^{*}\left(p_{2}\right) a_{\downarrow}\left(p_{3}\right) a_{\uparrow}\left(p_{4}\right) .
\end{aligned}
$$

The remaining part of the action we will label the molecular action

$$
\begin{aligned}
S_{M}= & \sum_{q, j}\left(i v-\frac{q^{2}}{4 m}-\nu_{j}+2 \mu\right) b_{j}^{*}(q) b_{j}(q) \\
& -\frac{1}{\sqrt{\beta V}} \sum_{q=p_{1}+p_{2}, j} g_{j}\left[b_{j}^{*}(q) a_{\downarrow}\left(p_{1}\right) a_{\uparrow}\left(p_{2}\right)\right. \\
& \left.+a_{\uparrow}^{*}\left(p_{2}\right) a_{\downarrow}^{*}\left(p_{1}\right) b_{j}^{*}(q)\right] .
\end{aligned}
$$

In deriving Eqs. (4) and (5) we have inserted contact potentials for the couplings $U\left(\boldsymbol{x}-\boldsymbol{x}^{\prime}\right) \rightarrow U \delta\left(\boldsymbol{x}-\boldsymbol{x}^{\prime}\right)$ and $g_{j}(\boldsymbol{x}$ $\left.-x^{\prime}\right) \rightarrow g_{j} \delta\left(x-x^{\prime}\right)$. The full partition function for our resonant system, under the model Hamiltonian of Eq. (2), can now be written as

$$
Z=\int\left(\prod_{\sigma} D a_{\sigma}^{*} D a_{\sigma}\right)\left(\prod_{j} D b_{j}^{*} D b_{j}\right) e^{S_{\mathrm{BCS}}+S_{M}},
$$

with the functional integral, $D c \equiv \Pi_{i} d c^{i}$, ranging over all Fermi and Bose fields.

\section{SADDLE-POINT APPROXIMATION}

From the form of the action in Eq. (6), it should be apparent that all of the resonant contributions are contained within the molecular action. In practice this gives rise to the integral of a displaced Gaussian that can be easily evaluated. After integrating out the molecular degrees of freedom, we are left with the partition function:

$$
Z=\left(\prod_{j} Z_{B_{j}}\left(q_{j}^{2} / 4 m+\nu_{j}-2 \mu\right)\right) \int D a_{\sigma}^{*} D a_{\sigma} e^{S_{\mathrm{BCS}^{\prime}}}
$$

Here $Z_{B_{j}}\left(q_{j}^{2} / 4 m+\nu_{j}-2 \mu\right)$ is a Bose partition function describing the formation of bound molecules and $S_{\mathrm{BCS}}$ is the BCS action with a potential that is now dependent on both thermal frequencies and momentum. The interaction potential in the BCS action is, therefore, modified in the presence of a Feshbach resonance in the following way:

$$
U \rightarrow U-\sum_{j} \frac{g_{j}^{2}}{q_{j}^{2} / 4 m+\nu_{j}-2 \mu-i v} .
$$

With the above partition function, Eq. (7), we may go on to calculate all thermodynamic properties of interest. Here, we are primarily interested in calculating the critical temperature of the superfluid phase transition. This can be done by solving for the gap and number equation, and then selfconsistently solving these two equations for both the chemical potential and the critical temperature. The procedure is straightforward since the full resonant calculation has been reduced to the usual BCS calculation, only with a more complicated potential. Following Popov's derivation [22], introducing the complex auxiliary Bose field $c(q)$ and expanding about the neighborhood of its zero value (which is equivalent to saying that we expand about the zero of the gap near $T_{c}$ ), we derive the gap equation at the critical point 


$$
1=\left(-U+\sum_{j} \frac{g_{j}^{2}}{\nu_{j}-2 \mu}\right) \sum_{k} \frac{\tanh \left[\beta\left(k^{2} / 2 m-\mu\right) / 2\right]}{2\left(k^{2} / 2 m-\mu\right)} .
$$

The second self-consistent equation, the number equation, is found in the saddle-point approximation by expanding the action to lowest order, i.e., $c(q)=c^{*}(q)=0$, and using the thermodynamic identity $N=-\partial \ln Z / \partial \mu$ giving

$$
N=2 \sum_{j, k} \frac{1}{e^{\beta\left(k^{2} / 4 m+\nu_{j}-2 \mu\right)}-1}+2 \sum_{k} \frac{1}{e^{\beta\left(k^{2} / 2 m-\mu\right)}+1} .
$$

Thus our number equation counts all free fermions, $N_{f}$, plus an additional boson population $N_{b}$. Equations (9) and (10) provide for us a set of equations for determining $T_{c}$ and $\mu$ at the critical point. In the usual BCS theory, this level of approximation proves reasonable for calculating $T_{c}$ in the BCS limit (small negative scattering length), but diverges as the scattering length grows, and is wholly inapplicable for positive scattering lengths. The reason for this is that the primary mechanism for the phase transition within the weak coupling BCS limit is the formation and disassociation of Cooper pairs. As the coupling increases the particles tend to pair up at higher and higher temperatures which means that the critical transition is no longer signaled by the formation of Cooper pairs, but rather by a coherence across the sample caused by condensation of preformed Cooper pairs. Since we are interested in describing the resonant system at all detunings, the equations that we have derived so far are insufficient because they do not account for this process. We should, therefore, next focus on how to more accurately incorporate atom pairing into our model.

\section{BEYOND THE SADDLE-POINT APPROXIMATION}

To account for fluctuations in the fermion field, we follow the method of Nozières and Schmitt-Rink [14] in its functional form as put forth by Randeria et al. [19]. This procedure will introduce a next order correction to the saddle point calculation of the previous section. By expanding the action to second order in $c(q)$, and then calculating the number equation in the same way as was done when deriving Eq. (10), we introduce an additional population into the equation. The action becomes

$$
S\left(c(q), c^{*}(q)\right)_{\mathrm{BCS}} \approx S_{\mathrm{BCS}^{\prime}}(0,0)+\sum_{q}|c(q)|^{2} \chi(q),
$$

where we have defined the auxiliary function $\chi(q)$ as

$$
\begin{aligned}
\chi(q)= & \left(U-\sum_{j} \frac{g_{j}^{2}}{\frac{q^{2}}{4 m}+\nu_{j}-2 \mu-i \omega}\right) \\
& \times \sum_{k} \frac{1-f\left(\epsilon_{(q / 2)+k}\right)-f\left(\epsilon_{(q / 2)-k}\right)}{\epsilon_{(q / 2)+k}+\epsilon_{(q / 2)-k}-i \omega} .
\end{aligned}
$$

Here $f\left(\epsilon_{k}\right)$ is the Fermi distribution function and $\epsilon_{k}=k^{2} / 2 m-\mu$. The resulting modified number equation is

$$
\begin{aligned}
N= & 2 \sum_{j, k} \frac{1}{e^{\beta\left(k^{2} / 4 m+\nu_{j}-2 \mu\right)}-1}+2 \sum_{k} \frac{1}{e^{\beta\left(k^{2} / 2 m-\mu\right)}+1} \\
& -\frac{1}{\beta} \sum_{\boldsymbol{q}, \omega} \frac{\partial}{\partial \mu} \log [1-\chi(\boldsymbol{q}, i \omega)] .
\end{aligned}
$$

This inclusion of the first order fluctuations introduces a population of atom pairs, $N_{p}$, that behave like bosons. We are now able to solve for the fluctuation corrected critical temperature from a self-consistent solution of Eqs. (9) and (13).

Due to the contact form of the couplings that we have chosen, however, we are immediately plagued with problems of divergences in our equations. This can be remedied by a proper renormalization, which means replacing the "bare" couplings and detunings by the correct renormalized forms depending on the actual physical parameters as well as a momentum cutoff $K_{c u t}$. This same procedure is needed to renormalize the usual, nonresonant BCS theory [21] and is done by relating the bare potential to the two-particle scattering matrix, $T$, through the Lippman-Schwinger equation. This leads to a renormalization of the coupling as $U=\Gamma \bar{U}$, where the bar notation represents a bare parameter. We make the definition $\alpha=m K_{\text {cut }} /\left(2 \pi^{2}\right)$ and introduce the dimensionless factor $\Gamma=(1-\alpha \bar{U})^{-1}$. This renormalization incorporates the true microscopic physics into the problem, removing the unphysical divergence.

For the resonant case, a more sophisticated approach is required, which we have extensively studied previously [10]. In the case of a single resonance (i.e., the $j$ sum has only a single term) the renormalization is performed by the following relations: $U=\Gamma \bar{U}, g=\Gamma \bar{g}$, and $\nu=\bar{\nu}+\alpha g \bar{g}$. The chemical potential of each atomic state is modified to $\mu=\bar{\mu}$ $-\left\langle T(k) n_{k}\right\rangle$ to include the proper mean-field shifts induced by all two-body scattering processes, where $n_{k}$ is the Fermi distribution, $T(k)$ is the two-body scattering matrix for the resonant system [10], and \langle\rangle denotes an averaging. This shift, however, is sufficiently small to neglect; inclusion has demonstrated corrections of the order of $1 \%$ or less. By replacing the bare values in Eqs. (9) and (13) with the renormalized values, all of the results to be discussed have been shown to be independent of the introduced momentum cutoff $K_{c u t}$.

The renormalization of the resonance theory forces us to take a closer look at the bound state physics of the system. In Fig. 1 we show the bound state energies for a single resonance system with a positive background scattering length. The figure results from a coupled square well calculation of the bound state energies [10] and shows the avoided crossing of two molecular states. The upper state behaves to a fairly good approximation as $E_{b}=\left(m a_{\text {eff }}^{2}\right)^{-1}$, which is the molecular binding energy regularly associated with a contact interaction [23]. The lower state, however, is offset from the detuning by an energy $\sim \kappa$ and goes linear with the detuning. We find a similar behavior as in the lower state in the first term of Eq. (13). Taking the cutoff to infinity, which is justified since this term does not diverge, the renormalized detuning approaches $\nu \rightarrow \bar{\nu}-\bar{g}^{2} / \bar{U}$. This produces a constant 


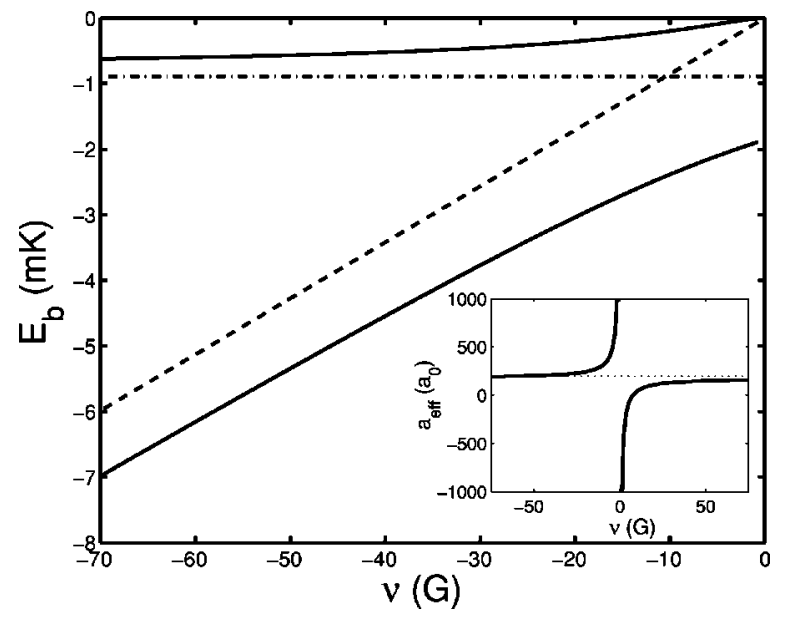

FIG. 1. Binding energies for ${ }^{40} \mathrm{~K}$ resonance at positive background scattering length $a_{\mathrm{bg}}=176 a_{0}$. A single resonance with a positive background scattering length produces an effective scattering length $a_{\mathrm{eff}}=a_{\mathrm{bg}}(1-\kappa / \nu)$, as seen in the inset where we plot the effective scattering length vs detuning (the dotted line is at $176 a_{0}$ ). A positive $a_{\mathrm{bg}}$, which is larger than the range of the potential, implies that another bound state is not far below threshold (dashdotted line). In combination with the Feshbach state (dashed detuning line) this results in an avoided crossing and the molecular state of interest asymptotes quickly to the dash-dotted line.

shift of $\bar{g}^{2} / \bar{U}=\kappa$ between the detuning and the molecular binding energy. Keeping this term in the number equation would incorrectly cause a transfer of the entire population into the wrong molecular state. In order to avoid this unwanted behavior we set this term to zero, i.e., $N_{b}=0$. In the case of a negative background scattering length, we would not have encountered this problem, and only one molecular state would have appeared (see Fig. 2). We will show in the next section that the pairing term fully accounts for the correct population of molecules in this system with $a_{\mathrm{bg}}>0$.

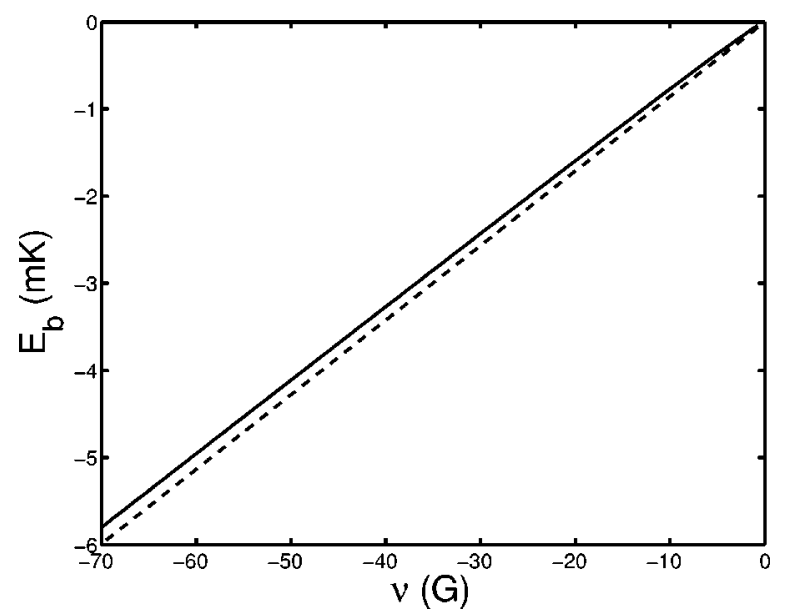

FIG. 2. Same as Fig. 1, but now for an artifical situation with $a_{\mathrm{bg}}<0$. A resonance system with a negative background scattering length has only one bound state relatively close to threshold, which is shifted to the positive of the detuning. The next bound state in the potential is too far away to be of any significant influence. The line styles are the same as for Fig. 1.
Before we present the full crossover solution for the case of ${ }^{40} \mathrm{~K}$, let us look at the analytical solutions to Eqs. (9) and (13) in the strong (BEC) and weak (BCS) coupling regimes. We will first turn our attention to the weak coupling (BCS) regime. In this limit we would expect only free fermions to contribute to the population, so from Eq. (13) we find that the chemical potential is at the Fermi surface $\left(\mu=E_{\mathrm{F}}\right)$. With this information, we solve the gap equation for the critical temperature. The result is the usual exponential dependence on the effective scattering length

$$
T_{c} / T_{F} \approx \frac{8}{\pi} e^{\gamma-2} \exp \left(\frac{-\pi}{2 k_{\mathrm{F}}\left|a_{\mathrm{eff}}\right|}\right),
$$

where $\gamma \sim 0.5772$ is the Euler-Mascheroni constant, $k_{\mathrm{F}}$ is the Fermi wave number, and $a_{\text {eff }}<0$ is the effective scattering length produced by the Feshbach resonance $a_{\mathrm{eff}}=a_{\mathrm{bg}}(1$ $-\kappa / \nu)$.

The other limit we may consider is the strong coupling (BEC) limit. When the argument of the tanh function in the gap equation (9) becomes sufficiently negative, it is a good approximation to use its asymptotic value of unity. What this means physically is that the fermion statistics are unimportant in determining the value of the gap. This allows us to solve the gap equation for the chemical potential as a function of detuning. In the limit of large negative detuning we find that $\mu \rightarrow-E_{b} / 2$, where $E_{b} \approx 1 / m a_{\text {eff }}^{2}$. Within this limit the entire population has been converted to molecules and we can solve the number equation to get the BEC condensation temperature of $T_{c} / T_{F} \sim 0.218$.

\section{NUMERICAL RESULTS}

To study the transition between the BEC and BCS regimes, we numerically solve Eqs. (9) and (13) for ${ }^{40} \mathrm{~K}$. The single resonance curve is produced using a background scattering length of $176 a_{0}$ and $\kappa=7.68 \mathrm{G}$ at a density of $10^{14} \mathrm{~cm}^{-3}$.

Figure 3 shows the critical temperature as a function of magnetic field detuning. The crossover calculation clearly merges with the BEC result for large-positive detunings and smoothly connects between positive and negative detunings, limiting to the Bose condensation temperature of $T_{c} / T_{F}$ $\sim 0.218$ for large negative detuning. This approach gives a maximum near zero detuning $\left(T_{c} / T_{F} \sim 0.26\right)$, but the maximum critical temperature we find is less than the predictions of the HFB approach in our earlier papers $\left(T_{c} / T_{F} \sim 0.5\right)$ [24]. We believe this is due to the inclusion of fluctuations in the beyond saddle point approximation which act to reduce the gap at zero temperature and therefore the critical temperature for the formation of a superfluid.

Figure 4 shows the chemical potential as a function of detuning, beginning at the Fermi energy for positive detuning and approaching half the bound state energy at large negative detuning $\mu \rightarrow-E_{b} / 2$. Figure 5 shows the change in population as a function of detuning. For large positive detuning, the system is composed solely of free fermions. As the detuning is decreased (i.e., from positive to negative) the contribution of the fermions begins to decrease until all the population is transferred into the atom pairs at $\nu \sim-0.5 \mathrm{G}$. 


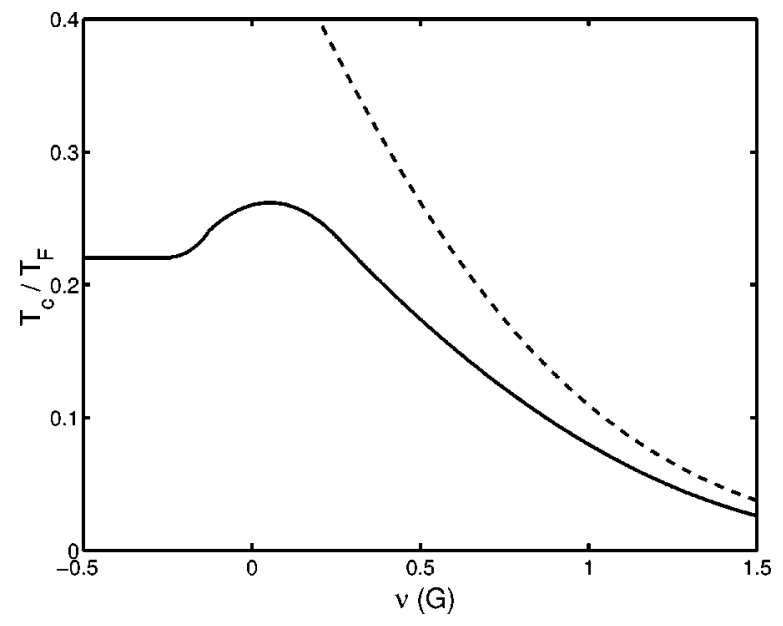

FIG. 3. Critical temperature $T / T_{F}$ as a function of detuning $\nu$ in gauss. The dashed line corresponds to the usual BCS solution, which limits to the full crossover theory at large positive detuning. At negative detuning, $T_{c}$ drops to the BEC condensation temperature of $T_{c} / T_{F} \sim 0.218$.

The chemical potential is then equal to $-E_{b} / 2$ and we may identify the atom pairs from that point as the molecules. The superfluid behavior then comes from the condensation of these molecules, which are no longer disassociating into free fermions.

\section{CONCLUSION}

We have presented a crossover model to describe the behavior of a gas of fermionic atoms for all detunings from a Feshbach resonance. The model is able to smoothly connect between the BCS and BEC regimes and accounts for the microscopic two-body physics throughout. We find a smooth behavior of the transition temperature in the entire crossover regime, with a maximum near zero detuning of $T_{c} / T_{f}$ $\sim 0.26$, and agreeing with the appropriate BEC and BCS behaviors in the respective large detuning limits. The maxi-

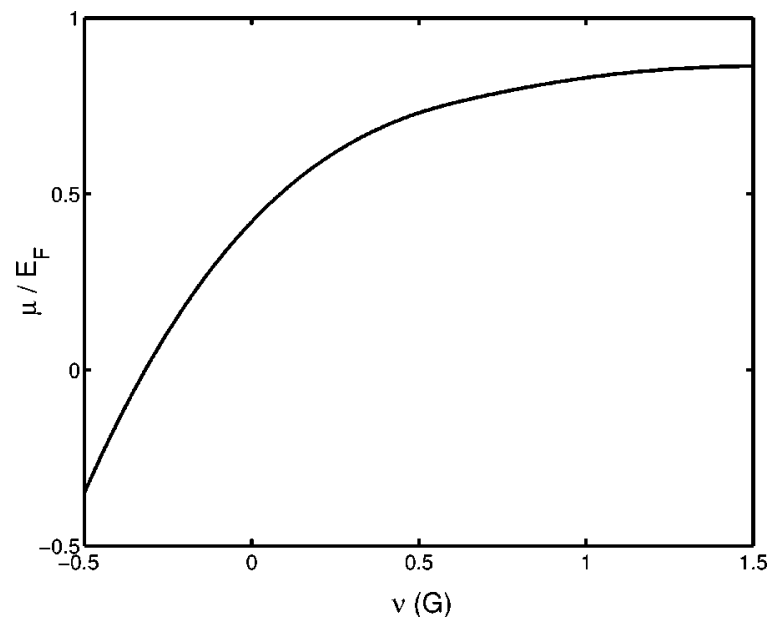

FIG. 4. Chemical potential as a function of detuning $\nu$ in gauss. For large negative detuning $2 \mu$ approaches the bound state energy of the molecular state. At increasing positive detuning, the chemical potential slowly approaches the Fermi energy.

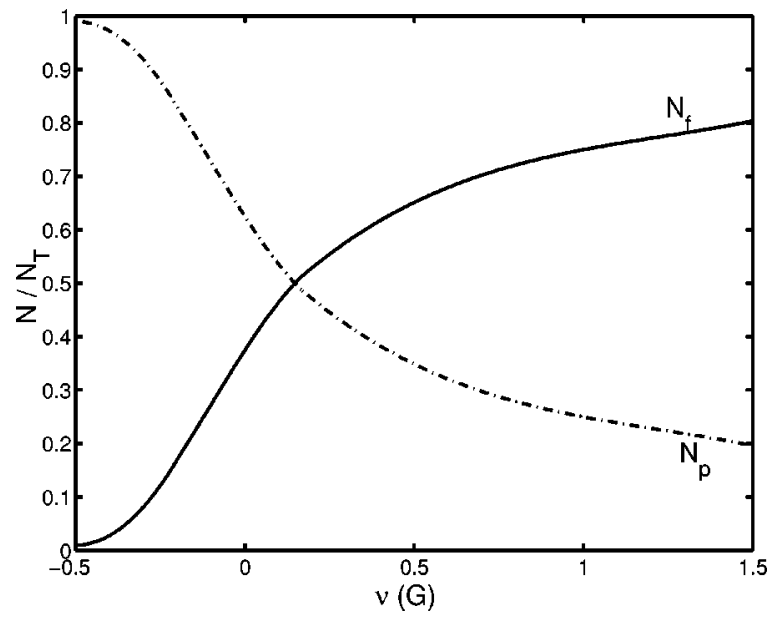

FIG. 5. The fraction of the total population as a function of detuning $\nu$ in gauss. The dot-dashed line corresponds to the pairing fraction $N_{p}$ and the solid to the free fermion fraction $N_{f}$.

mum is below the value predicted by the Hartree-FockBogoliubov theory for the uniform gas derived in earlier work. This result is a direct indication of the important role of preformed atom pairs which are neglected above $T_{c}$ in the Hartree-Fock-Bogoliubov theory. Such atomic pairing is represented as fluctuations in the fermion pairing field and modify the elementary excitation spectrum even in the normal phase. We emphasize, however, that in this paper we have only accounted for the pairing physics by including the second order fluctuations in order to be able to account for the correct molecular binding energy as derived from the two-body scattering physics. While the inclusion of this order of fluctuations is the main ingredient necessary to encapsulate essential aspects of the behavior of the system in the crossover regime, it would be interesting to extend the approach to consider the effect of higher order interactions which have not been accounted for. For example, we have only included interactions between free fermion atoms, neglecting all other contributions such as the interactions between pairs. This is most clearly seen in the BEC limit, where our solution adopts the thermodynamic behavior of the ideal Bose gas, rather than the dilute interacting Bose gas. A more sophisticated treatment could extend our approach to consider all these factors. Nevertheless, the results we have presented here illustrate the realistic potential in this realizable system for increasing the superfluid transition temperature, with the aid of the Feshbach resonance, to a significant fraction of the Fermi temperature. This is an important and timely aspect from a practical perspective because the maximum value we predict is in the region of the temperature range which is currently experimentally accessible.

\section{ACKNOWLEDGMENTS}

We would like to thank Yoji Ohashi for stimulating discussion on the numerical work. Support is acknowledged for J. M. and S. K. from the U.S. Department of Energy, Office of Basic Energy Sciences via the Chemical Sciences, Geosciences and Biosciences Division, and for M. H. from the National Science Foundation. 
[1] B. DeMarco and D.S. Jin, Science 285, 1703 (1999).

[2] F. Schreck, L. Khaykovich, K.L. Corwin, G. Ferrari, T. Bourdel, J. Cubizolles, and C. Salomon, Phys. Rev. Lett. 87, 080403 (2001).

[3] Z. Hadzibabic, C.A. Stan, K. Dieckmann, S. Gupta, M.W. Zwierlein, A. Görlitz, and W. Ketterle, Phys. Rev. Lett. 88, 160401 (2002).

[4] G. Truscott, K.E. Strecker, W.I. McAlexander, G.B. Partridge, and R.G. Hulet, Science 291, 2570 (2001).

[5] G. Roati, F. Riboli, G. Modugno, and M. Inguscio, e-print cond-mat/0205015.

[6] S.R. Granade, M.E. Gehm, K.M. O'Hara, and J.E. Thomas, Phys. Rev. Lett. 88, 120405 (2002).

[7] H.T.C. Stoof, M. Houbiers, C.A. Sackett, and R.G. Hulet, Phys. Rev. Lett. 76, 10 (1999).

[8] R. Combescot, Phys. Rev. Lett. 83, 3766 (1999).

[9] H. Heiselberg, C.J. Pethick, H. Smith, and L. Viverit, Phys. Rev. Lett. 85, 2418 (2000).

[10] S.J.J.M.F. Kokkelmans, J.N. Milstein, M.L. Chiofalo, R. Walser, and M.J. Holland, Phys. Rev. A 65, 053617 (2002).

[11] Gorkov finds, by including fluctuations within a higher order diagrammatic calculation, a factor of approximately 2 reduction in $T_{c}$ within the BCS regime [12].

[12] L.P. Gorkov and T.K. Melik-Barkhudarov, Sov. Phys. JETP 13, 1018 (1961).

[13] Y. Ohashi and A. Griffin, Phys. Rev. Lett. 89, 130402 (2002).

[14] P. Nozières and S. Schmitt-Rink, J. Low Temp. Phys. 59, 195 (1985).
[15] D.M. Eagles, Phys. Rev. 186, 456 (1969).

[16] A.J. Leggett, in Modern Trends in the Theory of Condensed Matter, edited by A. Pekalski and J. Przystawa (Springer, Berlin, 1980).

[17] F. Pistolesi and G.C. Strinati, Phys. Rev. B 49, 6356 (1994); S. Stintzing and W. Zwerger, ibid. 56, 9004 (1997).

[18] R. Haussmann, Z. Phys. B: Condens. Matter 91, 291 (1993); R. Haussmann, Phys. Rev. B 49, 12975 (1994).

[19] C.A.R. Sa de Melo, M. Randeria, and J.R. Engelbrecht, Phys. Rev. Lett. 71, 3202 (1993); for a review, see M. Randeria, in Bose-Einstein Condensation, edited by A. Griffin, D.W. Snoke, and S. Stringari (Cambridge University Press, Cambridge, England, 1995).

[20] E. Tiesinga, B.J. Verhaar, and H.T.C. Stoof, Phys. Rev. A 47, 4114 (1993).

[21] A.A. Abrikosov, L.P. Gorkov, and I.E. Dzyaloshinkski, Methods of Quantum Field Theory in Statisitical Physics (Dover, New York, 1963).

[22] V.N. Popov, Functional Integrals and Collective Excitations (Cambridge University Press, Cambridge, England, 1990), p. 204.

[23] R. G. Newton, Scattering Theory of Waves and Particles (McGraw-Hill, New York, 1966).

[24] M.L. Chiofalo, S.J.J.M.F. Kokkelmans, J.N. Milstein, and M.J. Holland, Phys. Rev. Lett. 88, 090402 (2002); M. Holland, S.J.J.M.F. Kokkelmans, M.L. Chiofalo, and R. Walser, ibid. 87, 120406 (2001). 